\title{
Psychophysiological Measurement of Personality
}

\section{Eco de Geus}

Vrije Universiteit, Amsterdam, The Netherlands

\author{
David Neumann \\ Griffith University, Gold Coast, Queensland, Australia
}

\section{INTRODUCTION}

Whereas the environment is in constant flux and causes changes in an individual's behavior over time, personality is considered the constant factor that causes stability in behavior. This constancy is likely to be hard-wired in our biology, and the brain seems the obvious place for such hard-wiring. Can we measure the exact shape of all this hard-wiring of behaviour? Is there a biological signal that closely predicts feelings and behavior and could be used as an "objective" tool to measure personality?

In this chapter we review attempts to correlate personality traits to individual differences in central nervous system functioning using psychophysiological recording techniques. This relatively small literature has been motivated by two different goals. The first has emerged from concerns about the validity of paper-and-pencil personality assessment (Eisenberger, Lieberman, \& Satpute, 2005). Virtually all major personality inventories are based on potentially flawed subjective linguistic self-report. To further advance personality testing, it may be necessary to move to objective tests (that may or may not involve use of words) that avoid most of the motivational and response distortion associated with item transparency of self-report 
instruments. Psychophysiological testing seems a promising method to do so, because voluntary control over the recorded biological signals is limited if not absent. A second motive to use psychophysiological testing in personality research is to elucidate the biological processes underlying the major dimensions of personality. Several influential models of personality, such as those by Eysenck (Eysenck, 1990a; Eysenck, 1967a), Gray (Gray, 1982; Gray, 1970b; Gray, 1971; Gray \& McNaughton, 2000b), Cloninger (Cloninger, Przybeck, \& Svrakic, 1991; Cloninger, Svrakic, \& Przybeck, 1993; Cloninger, 1986), and Zuckerman (Zuckerman, 1979; Zuckerman, 1991; Zuckerman \& Cloninger, 1996) have been strongly informed by biological theory, in that individual variation in the efficacy of various neurotransmitter systems, size and interconnectivity of neural structures mediating trait-typical behavior is a central construct in these theories. Psychophysiological testing can be used to corroborate these hypothesized biological correlates of personality, and ultimately chart the various intermediate steps in the biological pathways connecting variation in brain function to variation in behavior. 